\title{
Case of Rare Abdominal Wall Hernia
}

\author{
Aleksandr A. Reznichenko
}

\begin{abstract}
Posterior rectus sheath hernias are extremely rare. Exact mechanism of these hernias is not well understood. These hernias located between posterior rectus sheath and rectus muscle, above or below the umbilicus. As any other ventral hernias, posterior rectus sheath hernias may be presented as an emergency. US and/or CT may be helpful to suggest the posterior rectus sheath hernia before exploration. Diagnosis is made in surgery.
\end{abstract}

Keywords: Posterior rectus sheath hernia; Abdominal wall; Diagnosis

\section{Introduction}

The rectus sheath is described as consisting of anterior and posterior layers (lamella) formed by the aponeuroses of the external and internal oblique and transversus abdominis muscles. These aponeuroses meet at the lateral edge of the rectus along a curved line termed the linea semilunaris, which extends from the ninth costal cartilage to the pubic tubercle. Through most of the abdomen, the aponeuroses divide to pass around either side of the rectus muscle creating an anterior and posterior sheath. The anterior sheath is comprised of the aponeurosis of the external oblique and an anterior layer of the aponeurosis of internal oblique; the posterior sheath of the posterior layer of the internal oblique and the aponeurosis of the transversus abdominis muscle. Inferior to the plane that is located approximately halfway between the umbilicus and the symphysis pubis, all three aponeuroses pass anterior to the rectus muscle. This anterior displacement

Manuscript accepted for publication August 18, 2014

Tulare Regional Medical Center, 869 N Cherry St, Tulare, CA 93274, USA. Email: areznik9@yahoo.com

doi: http://dx.doi.org/10.14740/jcs238w of the aponeuroses creates a crescentic line of demarcation in the posterior lamella of the rectus sheath called the arcuate line, below which only the transversalis fascia separates the rectus abdominis muscle from the parietal peritoneum. Hernias of posterior rectus sheath are very rare. They represent so called interparietal hernias in which the hernia sac lies between the various layers of the abdominal wall. The exact mechanism of these hernias is not well understood. Since it is a rare condition, it is generally reported as sporadic cases and only eight cases have been reported in the literature. We present one case of posterior rectus sheath hernia.

\section{Case Report}

A 39-year-old female presented with $10 \mathrm{~h}$ of painful bulge in right lower quadrant and several episodes of nausea and vomiting. She had h/o lap cholecystectomy, two c-sections, back surgery, HTN and chronic back pain. She was an active smoker. Physical exam showed a painful mass in right lower guadrant with local rebound tenderness. She had a lower abdominal wall scar after c-section. A CT scan of the abdomen and pelvis showed abdominal wall hernia with loops of small bowel extending into the right rectus abdominis muscle (Fig. 1, 2). Pt was immediately taken to the OR. Right paramedian incision $8 \mathrm{~cm}$ long was performed above palpable mass, anterior rectus sheath was opened, approximately $65 \mathrm{~cm}$ loop of small bowel was strangulated and necrotic in hernia sac

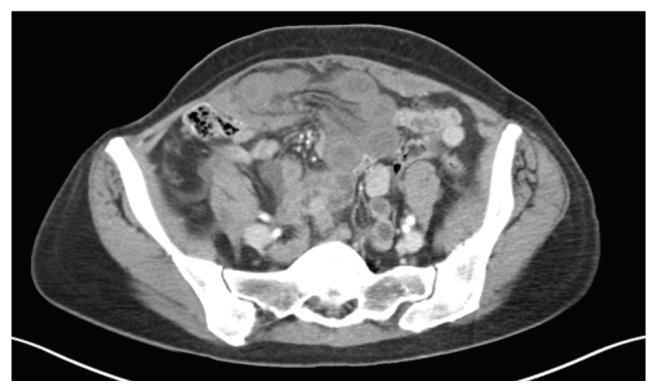

Figure 1. CT scan of the patient (axial view) showing herniation of the small bowel in between rectus sheath and rectus muscle on the right side. 


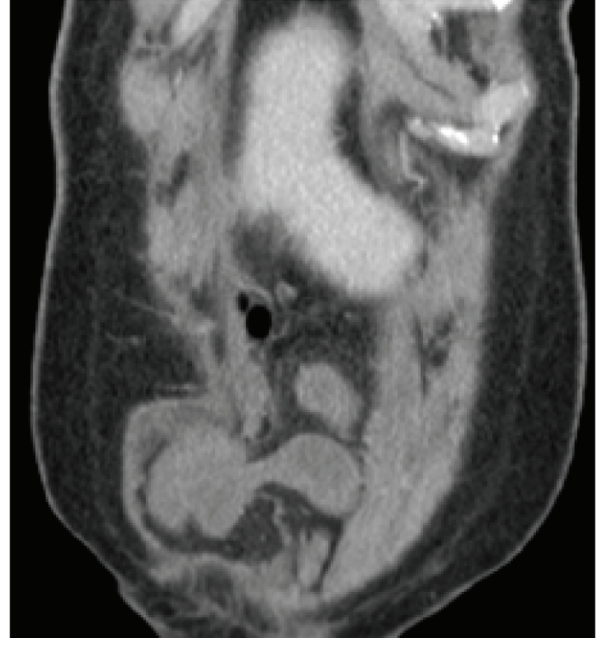

Figure 2. CT scan of the patient (coronal view) showing rectus sheath hernia.

located between posterior rectus sheath and rectus muscle. Rectus muscle was inflamed. Small bowel resection with primary anastomosis was performed. Posterior rectus sheath and rectus muscle were closed with running PDS \#1, anterior rectus sheath was closed with interrupted figure of 8 vicryl $\# 1$. Pt made uneventful recovery and was discharged home on POD \#5.

\section{Discussion}

Hernias of the posterior rectus sheath are rare [1]. Spontaneous ventral hernia through the rectus abdominis sheath is perhaps the rarest hernia, with eight previously reported cases since 1937 [2]. It belongs to so called interparietal hernias in which the hernia sac lies between the various layers of the abdominal wall [3]. These hernias mainly are described in the inguinal region and are divided into three categories, of which the interstitial type is the most frequent [3]. In that group, the hernia sac lies between the muscle layers of the abdominal wall. This description fits the clinical picture of the hernia of the posterior sheath of the rectus abdominis muscle because it does not protrude beyond this layer [4].

Despite that the exact mechanism of these hernias is not well known, some authors have emphasized the role of predisposing factors known in all kinds of hernias, mainly increased muscle weakness and elevated intraabdominal pressure as during pregnancy and in cases of ascites $[1,4]$.

The rectus sheath encloses the rectus abdominis muscle and is formed by the aponeurosis of the flat abdominal muscles. Its anterior layer consists of the external oblique aponeurosis supplemented by the anterior aponeurotic layer of the internal oblique aponeurosis, whereas its posterior layer is formed by the aponeuroses of the transversus abdominis muscle and the posterior aponeurotic layer of the internal oblique aponeurosis up to the level of the arcuate line. However, below the arcuate line, it is reduced to the fascia transversalis because all three aponeuroses pass anterior to the rectus abdominis muscle [5]. Although strong, the rectus sheath shows sites of minor resistance susceptible to explain these hernias without previous traumatic or surgical history [4].

Posterior rectus sheath hernias could be considered before surgery with either US [1, 4] or CT [6], like in our case. However, they were finally diagnosed during surgery [1].

Our case is notable because hernia was located in infraumbilical region, in the contrary to all other reported cases, where it was presented above the umbilicus [6].

In conclusion, we present a case posterior rectus sheath hernia. A surgeon should be aware of this very rare type of hernia, and may be able to suspect it preoperatively on imaging studies. Principles of surgical repair of these hernias are the same as other types of ventral hernias.

\section{Acknowledgement}

None.

\section{References}

1. Bentzon N, Adamsen S. Hernia of the posterior rectus sheath: a new entity? Eur J Surg. 1995;161(3):215-216.

2. Losanoff JE, Basson MD, Gruber SA. Spontaneous hernia through the posterior rectus abdominis sheath: case report and review of the published literature 1937-2008. Hernia. 2009;13(5):555-558.

3. Etawo US, Elechi EN. Interparietal hernias: analysis of six cases with literature review. Br J Clin Pract. 1987;41(12):1068-1070.

4. Gangi S, Sparacino T, Furci M, Basile F. Hernia of the posterior lamina of the rectus abdominis muscle sheath: report of a case. Ann Ital Chir. 2002;73(3):335-337.

5. Flament JB, Avisse C, Delattre JF. Anatomy of the abdominal wall. In: Bendavid R, Abrahamson J, Arregui $\mathrm{M}$, et al., eds. Abdominal wall hernias, principles and management. Heidelberg, Germany: Springer-Verlag; 2001:41-42.

6. Mounia F, Mona EK, Yves M, Didier S, Yves M. Incarcerated Hernia through the Posterior Rectus Sheath. Gastrointestinal Imaging. 2005;185(5). 